\title{
Ineffability within the Limits of Abstraction Alone
}

\author{
Stewart Shapiro and Gabriel Uzquiano
}

\section{Abstraction and iteration}

The purpose of this article is to assess the prospects for a Scottish neo-logicist foundation for a set theory. The gold standard would be a theory as rich and useful as the dominant one, ZFC, Zermelo-Fraenkel set theory with choice, perhaps augmented with large cardinal principles. Although the present paper is self-contained, we draw upon recent work in [32] in which we explore the power of a reasonably pure principle of reflection.

To establish terminology, the Scottish neo-logicist program is to develop branches of mathematics using abstraction principles in the form:

$$
\S \alpha=\S \beta \leftrightarrow \alpha \sim \beta
$$

where the variables $\alpha$ and $\beta$ range over items of a certain sort, $\S$ is an operator taking items of this sort to objects, and $\sim$ is an equivalence relation on this sort of item.

The standard exemplar, of course, is Hume's principle:

$$
\# F=\# G \equiv F \approx G
$$

where, as usual, $F \approx G$ is an abbreviation of the second-order statement that there is a one-one correspondence from $F$ onto $G$. Hume's principle, is the main support for what is regarded as a success-story for the Scottish neo-logicist program, at least by its advocates. The search is on to develop more powerful mathematical theories on a similar basis.

As witnessed by this volume and a significant portion of the contemporary literature in the philosophy of mathematics, Scottish neo-logicism remains controversial, even for arithmetic. There are, for example, issues of Caesar and Bad Company to deal with. Here, we will only touch briefly on these problems, and only to the extent to which they bear on the prospects for a candidate neo-logicist set theory. We take the success of the program for arithmetic for granted, at least for 
the sake of argument. We will also concede that there is, or can be, an adequate neologicist theory of real analysis — perhaps along the lines of [11] or [29]—complex analysis, functional analysis, and the like. Our focus is on the prospects for a neologicist set theory.

The standard motivation for the axioms of set theory stems from the technical observation that Zermelo-Fraenkel set theory, with or without choice, $\mathrm{ZF}(\mathrm{C})$, proves that every set is a member of some $V_{\alpha}$, where, as usual:

$$
V_{0}=\emptyset ; V_{\alpha+1}=\mathcal{P}\left(V_{\alpha}\right) ; \text { and } V_{\lambda}=\bigcup_{\alpha<\lambda} V_{\alpha} \text { for limit ordinals } \lambda,
$$

where $\emptyset$ is the empty set and for each set $x, \mathcal{P}(x)$ is the power set of $x$. This description of the cumulative hierarchy of well-founded sets dates back (at least) to [22], [35], and [39]. ${ }^{1}$ But while the early fathers of set theory sometimes conceived of this cumulative hierarchy as an inner model of the axioms, the iterative picture of the universe of set theory as layered in levels or stages eventually emerged with Kurt Gödel as a powerful heuristic for motivating the axioms of set theory generally. This is what has come to be known as the iterative conception of set.

When put this way, the cumulative hierarchy seems to presuppose the ordinals. However, ordinals are generally taken to be von Neumann ordinals which, of course, are certain sets - transitive pure sets well-ordered by membership. The picture that emerges is that there is a stage corresponding to every von Neumann ordinal, and there is a von Neumann ordinal corresponding to each stage. ${ }^{2}$

Many of the axioms of ZFC can be viewed as assumptions concerning the extent of the cumulative hierarchy. They set lower bounds on the extent of the ordinal spine, which, in turn, determines the extent of the hierarchy. For example, the axiom of infinity amounts to the existence of a first transfinite stage, $V_{\omega}$. The power set axiom allows us to move from a given stage, $V_{\alpha}$, to its successor stage, $V_{\alpha+1}$, and therefore tells us that the universe itself resembles a "limit stage", which cannot be reached from below by the power-set operation. Even replacement can be regarded as a cofinality principle on the extent of the cumulative hierarchy, one designed to make sure that any set-sized collection of stages is bounded by some later stage of the cumulative hierarchy. ${ }^{3}$

One of the purposes of set theory is foundational: to provide surrogates for all mathematical objects, and thus an arena in which relations between mathematical theories can be investigated, questions of existence and coherence can be adjudi-

\footnotetext{
${ }^{1}$ See, for example, [17].

${ }^{2}$ Not every exposition of the cumulative hierarchy or the iterative conception presupposes the ordinals. [27] provided an axiomatization of set theory directly motivated by the picture of the universe of set theory as layered in levels—or "partial universes" as he called them-without explicit recourse to ordinals. Boolos' exposition of the iterative conception in [2] draws on this work as do, for example, more recent axiomatizations of iterative set theory in [24].

${ }^{3}$ See [3] and [2] for discussion.
} 
cated, etc. For better or worse, ZFC takes us well beyond what is needed to recapture most of ordinary mathematics, except of course foundational theories like category theory and set theory itself. Even without invoking sophisticated coding techniques, it is hard to imagine a need to go beyond, say, stage $V_{\omega+10}$. So even the weaker Zermelo set theory, which, on its common interpretation, takes the iteration up to (but not including) stage $V_{\omega+\omega}$, is more than sufficient for foundational purposes, at least for non-foundational mathematical theories. Thus, it would be quite a coup if even a theory as powerful as Zermelo set theory could be developed along neo-logicist lines. Then the neo-logicist could proudly recapitulate, or develop surrogates for, pretty much every standard mathematical theory, except perhaps for foundational ones. Given the epistemological goals of the neo-logicist program, there would still be questions concerning the relationship between the set-theoretic surrogates and the theories they are surrogates for. Can we account for a priori knowledge of the basic principles of mathematical theory $X$ by showing to derive a standard model for $X$ in a neo-logicist set theory? We hereby set all such Caesar-type questions aside.

While we are aiming high, notice that even full ZFC fails to completely satisfy common assumptions underlying the extent of the cumulative hierarchy. Most practicing set theorists do not take the axioms, in toto, to set an upper-bound on iteration. A cardinal $\kappa$ is strongly inaccessible if it is regular and larger than the powerset of any smaller cardinal. The axioms of ZFC do not entail the existence of any strongly inaccessible cardinals. Indeed, if $\kappa$ is a strong inaccessible, then the result of iterating the hierarchy to stage $\kappa$ will result in a model of ZFC (and this model satisfies the statement that there are no strongly inaccessible cardinals). Nevertheless, the existence of (lots of) strong inaccessibles is a staple of set theory. ${ }^{4}$ Indeed, strong inaccessibles are the very bottom of a scale of "small" large cardinals.

\section{Ineffability and reflection}

One common way to capture the assumption that there is no upper-bound on the iteration involved in the cumulative hierarchy-beyond platitudes such as that the iteration proceeds "all the way" or "as far as possible" or "through all the ordinals" is the admittedly vague thought that the universe of set theory is, in a sense to be elucidated, ineffable or indescribable. And here we broach a central topic of this paper, the extent to which this ineffability is compatible with Scottish neo-logicism.

The thought that the universe of set theory is ineffable has been exploited to motivate lower-bounds on the extent of the cumulative hierarchy, through what are

\footnotetext{
${ }^{4}$ An exception is Abraham Fraenkel's short lived axiom of restriction which asserts, in effect, that there are no sets other than those whose existence follows from directly from the other axioms. This is an analogue of the principle of induction in arithmetic. See Fraenkel [1922] and [9], 113-116.
} 
called "reflection principles". These principles tell us that no formula of a given language is able to uniquely characterize the universe of all sets. That is, if it is satisfied by the universe of all sets, then it is satisfied when suitably relativized to the members of some set. The strength of such principles is generally tied to the expressive resources of the language in question.

One heuristic motivation for these principles proceeds via a "limitation of size" theme, which is sometimes combined with the iterative conception in order to motivate the axioms of ZFC. Roughly, limitation of size is the thought that some objects form a set just in case they are not too many of them. But then one should say how many are too many. The admittedly vague Cantorian idea is that some sets are too many only if they are indeterminately or indefinitely many. The connection between this thought and reflection is made explicit by [4] and [5] (see ch. 3), with the following train:

(1) ... the sets are indeterminately or indefinitely many.

(2) ... the sets are indefinably or indescribably many.

(3) . . . any statement $\Phi$ that holds of them fails to describe how many they are.

(4) ... any statement $\Phi$ that holds of them continues to hold if reinterpreted to be not about all of them but just about some of them, fewer than all of them.

(5) ... any statement $\Phi$ that holds of them continues to hold if reinterpreted to be not about all of them but just some of them, few enough to form a set.

As Burgess notes, these steps are not meant to provide a deductive argument supporting reflection. All the same, reflection seems to be in line with the practice of set theorists.

In informal language, [4] indicates how to derive principles of set existence from (4):

. . . though it is possible to make a true statement about how many objects there are, there are too many objects for it to be possible for such a statement to be definitive of how many there are: there will necessarily be not merely as many as is said, but more also . . . To begin with, there is at least one object. By [(4)], that would still be true if one were speaking not of all objects, but just some of the objects, fewer than all; in other words, it is an understatement, which means that there must be at least two objects. Then, by [(4)] again, that is an understatement, so there are at least three objects. Continuing in this way, there are infinitely many objects. But by [(4)], even that is an understatement, so there must be uncountably many objects. But by [(4)] again, even that is an understatement, so . . (p. 192)

It turns out, however, that it is step (5) that provides the most power when we let 
$\Phi$ range over formulas of the language of higher-order set theory. ${ }^{5}$ For example, in the language of second-order ZFC, we can express the statement $\Phi$ that the universe of set theory, $V$, forms a strongly inaccessible class, and, since it is presumed that the universe is ineffable, $\Phi$ cannot characterize the universe uniquely. Therefore, there is a set in the cumulative hierarchy that satisfies $\Phi$. Thus, reflection entails the existence of an inaccessible cardinal.

In an early articulation of the iterative conception, [39] proposed the existence of "an unbounded sequence of [inaccessible cardinals] as a new axiom of meta-set theory." According to this principle, for each ordinal $\alpha$, there is a unique inaccessible cardinal $\kappa_{\alpha}$. But this is not the end of the process of reflection. Reflection on the new axiom would entail that there is a set $x$ such that for every ordinal $\alpha \in x$, $\kappa_{\alpha} \in x$. This, in turn, entails the existence of a fixed point in the $\kappa$-series: an ordinal $\kappa$ such that $\kappa=\kappa_{\kappa}$. This is called a "hyper-inaccessible". Successive applications of second-order reflection soon yield Mahlo cardinals of all orders, weakly compact cardinals and, more generally, $\Pi_{n}^{1}$-indescribable cardinals for each $n \in \omega .^{6}$ However, second-order reflection is still relatively weak by the lights of large cardinal axioms and lies at the bottom of an entire hierarchy of stronger and stronger indescribable cardinals which are known to be compatible with $V=L{ }^{7}$

\section{Now, Zermelo wrote:}

If we now put forward the general hypothesis that every categorically determined domain can also be interpreted as a set in some way, i.e., can appear as an element of a normal domain [a set-theoretic model of set theory], it follows that to each normal domain there is a higher domain with the same basis. ${ }^{8}$

${ }^{5}$ When we restrict ourselves to first-order formulas, every instance of (5) is a theorem of firstorder ZFC. In fact, this is the main observation [23] exploited to show that first-order ZFC is not finitely axiomatizable.

${ }^{6}$ In general, second-order reflection amounts to $\Pi_{n}^{1}$-indescribability for every $n \in \omega$. See [31] and [30], section 6.3. By a result due to Hanf and Scott, weakly compact cardinals are exactly the $\Pi_{1}^{1}$-cardinals. For details, see for example [18], Theorem 6.4, or [16], Theorem 17.18.

${ }^{7}$ That $\Pi_{n}^{m}$-indescribability is compatible with $V=L$ is noted, for example, by [18], Ch. 1 , Theorem 6.6. One has to proceed with caution when formulating reflection in terms of third- and higher-order languages. [25] observes that inconsistency results if we allow third-order parameters unrestrictedly. However, [33] has motivated a restriction of reflection with third- and higher-order parameters to a special class of formulas, i.e., positive formulas, and he has proposed a corresponding hierarchy of reflection principles. These principles take us well beyond $\Pi_{m}^{n}$-indescribability—or even beyond reflection on formulas of transfinite order without third- or higher-order parameters-as they lead to ineffable cardinals. Much stronger indescribability principles-some of which reflect formulas of transfinite order (with no third- or higher-order parameters) - fall under the consistency result proved in [19]. Koellner has proved a dichotomy theorem to the effect that generalized reflection principles, which include Tait's reflection principles, are either weak (i.e. consistent relative to $\kappa(\omega)$ and hence with $V=L$ ) or inconsistent. In the concluding section 9 of the present paper, we do briefly countenance ascent to higher-order principles of reflection, but we restrict attention to sentences, with no parameters of any order.

${ }^{8}$ See [39], 1232. 
In [36] (see p. 555), Hao Wang captured the idea succinctly:

Any time we try to capture the universe from what we positively possess (or can express) we fail the task and the characterization is satisfied by certain (large) sets.

\section{Framework}

[32] presents a reasonably pure version of ineffability and reflection, in the context of a Fregean theory of extensions. Without relying on any substantial set-theoretic assumptions concerning the structure of the set-theoretic universe, we develop a remarkably powerful theory, enough to show the consistency of Zermelo set theory with choice, and thus to recapture pretty much all extant mathematics, except possibly for foundational theories like category theory or set theory.

Here we briefly recapitulate our theory of extensions and reformulate one of its axioms in the shape of an abstraction principle. As we take ineffability and reflection to be an integral part of contemporary set theory, we then use this opportunity to assess the prospects for a neo-logicist set theory. On this score, our conclusions are mostly negative.

To the extent to which our abstraction principle will eventually yield much of ordinary mathematics, we think it may be of some interest for the neo-logicists. However, if they insist on ZFC as their target, then our result will still miss the mark by a long shot. Indeed, as we will eventually note, it is consistent with ZFC that our proposed abstraction has models of cardinality smaller than $\boldsymbol{\aleph}_{\omega_{1}}$, which is teensy even by the lights of ZFC itself, let alone any large cardinal principles.

We work in a second-order language, understood with full, standard semantics. We do not wish to make an issue over what the second-order variables range over: Fregean concepts, classes, logical sets, whatever. Since we will be concerned with a modification of Frege's Basic Law V, we will refer to the items in the range of the second-order quantifiers as Fregean concepts. But the reader should feel free to substitute a different locution if she so wishes.

Recall Frege's inconsistent Basic Law V:

$$
\epsilon(F)=\epsilon(G) \equiv \forall x(F x \equiv G x)
$$

Our plan is to begin with a version of Basic Law V which restricts the principle to certain concepts. Introduce a word GooD for those concepts, and let BAD stand for its complement.

There are several ways to restrict Basic Law V, with the choice between them only a matter of convenience. [32] employ a second-order predicate $\operatorname{ExT}(x, F)$, which is 
satisfied by an object $x$ and a concept $F$ just in case $x$ is an (or the) extension of $F$. Our axiom in [32] is thus:

$$
\operatorname{ExT}(x, X) \& \operatorname{Exт}(y, Y) \rightarrow(x=y \equiv \forall z(X z \equiv Y z))
$$

And we define GooD and BAD by means of the following:

$$
\begin{array}{lll}
\operatorname{Good}(F) & \equiv_{d f} & \exists x \operatorname{Ext}(x, F) \\
\operatorname{BAD}(F) & \equiv_{d f} & \neg \operatorname{Good}(F) .
\end{array}
$$

This, too, is the option [5] prefers.

A second option is to think of the extension operator $\epsilon$ as a partial function from GooD concepts to objects, in which case $\epsilon(F)$ becomes a non-denoting singular term when $F$ is BAD. We would then require a free logic to handle such terms. This is the option followed in [14] and [1]. Yet a third option is to think of the extension operator as a total function (i.e., defined on all concepts) but to indicate that we do not care what the "extension" of BAD concepts may be. ${ }^{9}$ Here we adopt yet a fourth option, followed by [2] and [28]. We take the extension operator to be total and assign a dummy object as the "extension" of all BAD concepts. Thus our principle is this:

$$
\epsilon(F)=\epsilon(G) \equiv((\mathrm{BAD}(F) \& \mathrm{BAD}(G)) \vee \forall x(F x \equiv G x)) .
$$

We now define a set and membership predicate in the obvious way. ${ }^{10}$

$$
\begin{array}{lll}
\operatorname{EXT}(x) & \equiv_{d f} & \exists X(\operatorname{Good}(X) \& x=\epsilon(X) \\
x \in y & \equiv_{d f} & \exists X(\operatorname{GooD}(X) \& y=\epsilon(X) \& X x) .
\end{array}
$$

Notice that (RV) has the form of an abstraction principle. [28] argues that any neo-logicist set theory would have to be based on a principle in a similar form. This is seconded by [10]. Moreover, (RV) will hold in just about any set theory imaginable. If we define a concept $F$ to be Good just in case there is a set whose members are all and only the instances of $F$, then (RV) is just the principle of extensionality.

On its own, however, (RV) is only a principle of extensionality. It does not follow from (RV) that there are any Good concepts, and thus any extensions at all. A set

${ }^{9}$ In this case, the relevant principle would be:

$$
\operatorname{Good}(F) \& \operatorname{Good}(G) \rightarrow(\epsilon(F)=\epsilon(G) \equiv \forall x(F x \equiv G x)) .
$$

\footnotetext{
${ }^{10}$ These definitions are slightly different from those in [2] and [28], where the restriction to GooD concepts is not invoked. Since the dummy "extension" of BAD concepts is in the range of the $\epsilon$ operator, those papers take it to be an "extension." Here we define the "EXT" predicate to exclude the dummy "extension", and we follow this in our informal gloss as well, concerning the English word "extension". In what follows, a phrase like " $X$ has an extension" is to be synonymous with " $X$ is Good”.
} 
theory will emerge only when we add some principles concerning which concepts are Good. One option, we suppose, would be to take GooD as a primitive and add the axioms of ZFC, phrased in those terms. The empty set axiom would be the statement that the empty concept is Good:

$$
\forall X(\forall x \neg X x \rightarrow \operatorname{GooD}(X)) ;
$$

the pair-set axiom would be:

$$
\forall x \forall y(\forall X(\forall z(X z \rightarrow(z=x \vee z=y))) \rightarrow \operatorname{GooD}(X)) ;
$$

and so forth. To belabor the obvious, the power, and the interest, of that theory would come from the added axioms, and have nothing to do with the neo-logicist program. Indeed, the theory is just a notational variant of ZFC, with the principle of extensionality formulated as an abstraction.

The best option for the neo-logicist would be to provide an explicit definition of GooD (or BAD), presumably using only logical resources, or perhaps logical resources augmented with other abstraction operators. This, we take it, is the crux of the problem. Although we do provide some explicit definitions below, our first plan is to use the notion of ineffability to motivate a reflection principle, which provides a sufficient condition for a concept to be GooD. This yields existence principles and a decent set theory (albeit still one weaker than ZFC). To foreshadow our conclusion, an explicit definition of Good (or BAD) would undermine the ineffability. It would be to characterize what the underlying motivation takes to be uncharacterizable or, to use a phrase coined in [32], to eff the ineffable. For now, then we take $\operatorname{Good}(X)$ to be a primitive of our system, and define $\operatorname{BAD}(X)$ as $\neg \operatorname{Good}(X)$.

\section{It's Goon is to be smaller than some concept describable in pure second-order logic}

We define the relativization of a sentence $\Phi$ to a concept $F$ to be the result of relativizing the quantifiers in $\Phi$ to $F$, by which we mean, as usual, the result of replacing:

$$
\begin{array}{lll}
\exists x(\ldots) & \text { with } \quad \exists x(F x \& \ldots) \\
\exists X(\ldots) & \text { with } \quad \exists X(\forall x(X x \rightarrow F x) \& \ldots) \\
\forall x(\ldots) & \text { with } \quad \forall x(F x \rightarrow \ldots) \\
\forall X(\ldots) & \text { with } \quad \forall X(\forall x(X x \rightarrow F x) \rightarrow \ldots) .
\end{array}
$$

We write $\Phi^{F}$ for the relativization of $\Phi$ to $F$. If the language in question has a relation for membership, and if $t$ is a term, then $\Phi^{t}$ is the relativization of $\Phi$ to the 
concept of being a member of $t$. That is, $\Phi^{t}$ is the result of replacing:

$$
\begin{array}{lll}
\exists x(\ldots) & \text { with } & \exists x(x \in t \& \ldots) \\
\exists X(\ldots) & \text { with } \exists X(\forall x(X x \rightarrow x \in t) \& \ldots) \\
\forall x(\ldots) & \text { with } \quad \forall x(x \in t \rightarrow \ldots) \\
\forall X(\ldots) & \text { with } \quad \forall X(\forall x(X x \rightarrow x \in t) \rightarrow \ldots) .
\end{array}
$$

If $F$ and $G$ are concepts, then let " $F \approx G$ " be the usual second-order statement that $F$ is equinumerous with $G$. That is, $F \approx G$ if and only if there is a one-toone relation from $F$ onto $G .{ }^{11}$ Similarly, let $F \leq G$ say that there is a one-to-one relation from $F$ to $G$, and let " $F<G$ " say that $F \leq G$ but not $G \leq F$.

If a sentence $\Phi$ with no non-logical terminology is true in a structure $\mathcal{M}$, then $\Phi$ is true in any structure whose universe is equinumerous with that of $\mathcal{M}$. In effect, the only distinctions among models that can be made with such "pure" sentences are differences of cardinality. Since we are concerned with relative sizes of various concepts and extensions, it makes sense to invoke purely logical sentences in reflection here.

Let $\Phi$ be a sentence in a formal language. The size a concept $F$ must have, in order for $\Phi^{F}$ to hold, is a lower bound that $\Phi$ imposes on the universe. For example, the sentence $\exists x \exists y(x \neq y)$ imposes a lower bound of 2 on the size of the universe, since $(\exists x \exists y(x \neq y))^{F}$ holds only if $F$ applies to at least two objects. And if PA2 is the conjunction of the axioms of second-order Peano arithmetic, then PA2 sets a lower bound of $\boldsymbol{\aleph}_{0}$ on the universe. Similarly, Z2, the conjunction of the axioms of second-order ZFC, sets a lower bound at the first strong inaccessible.

More formally, say that a concept $F$ is fixed by a sentence $\Phi$ just in case $\Phi^{F}$ and for every concept $X$, if $\Phi^{X}$ then $F \leq X$. This is at least one formal analogue of the notion of $\Phi$ setting a bound (on the universe) at the "size" of $F$. Say that $F$ is bounded if there is a sentence $\Phi$ of pure second-order logic (i.e., $\Phi$ has no non-logical terminology) and a concept $G$ such that $F \leq G$ and $G$ is fixed by $\Phi .{ }^{12}$

The thesis here is that every bounded concept is GooD. So for each sentence $\Phi$ of pure second-order logic, the following should hold:

$$
\exists G\left(F \leq G \& \Phi^{G} \& \forall H\left(\Phi^{H} \rightarrow G \leq H\right)\right) \rightarrow \operatorname{GooD}(F) .
$$

The schema seems to correspond to Burgess' (4) above. Our theory is hereby dubbed FZBB2, for Frege-Zermelo-Bernays-Burgess. ${ }^{13}$ It is axiomatized by the

\footnotetext{
${ }^{11}$ The formal definition of equinumerosity is well-defined on any pair of concepts, whether or not they have extensions.

${ }^{12}$ This notion of boundedness is a counterpart, in pure logic, of the model-theoretic notion of an ordinal being "pinned down" by a sentence.

${ }^{13}$ The "2" is to contrast the present system from FZBB, developed in [32]. That theory is axiomatized by a counterpart to (RF) and the analogue of the extensionality principle given above. FZBB can be interpreted in FZBB2 by defining $\operatorname{ExT}(x, X)$ as $(\operatorname{Good}(X) \& x=\epsilon(X))$. One can also interpret
} 
schema $(\mathrm{RF})$ and the aforementioned principle of extensionality:

$$
\epsilon(F)=\epsilon(G) \equiv((\mathrm{BAD}(F) \& \operatorname{BAD}(G)) \vee \forall x(F x \equiv G x)
$$

Although the intuitive thought is that all BAD concepts are concepts larger than any concept fixed by a sentence of pure second-order logic, we will eventually attend to higher-order generalizations of this. But notice how the restriction to closed sentences of the language enables us to stay clear from the specter of Reinhardt's observation, mentioned in footnote 7 , that higher-order reflection principles in which third-and higher-order parameters are allowed unrestrictedly lead to inconsistency.

\section{What we can offer}

Further details for the following results are in [32] (see note 12). FZBB2 entails the following:

Empty extension: $\exists x(\operatorname{EXT}(x) \& \forall y(\neg y \in x))$

Let $F$ be the empty concept $[x: x \neq x]$. Then $F$ is fixed by the sentence $\forall x(x \neq x)$ (which, of course, is vacuously satisfied by every concept). That is, we have $\forall x(x \neq x)^{F}$ and $\forall G\left(\forall x(x \neq x)^{G} \rightarrow F \leq G\right)$. So $F$ is bounded, and, by (RF), $F$ is Good. So $F$ has an extension, which we dub $\emptyset$.

Note, incidentally, the use of a neo-logicist ploy of beginning with the concept "non-self-identical". The bootstrapping continues:

Singletons: $\forall x \exists y(\operatorname{EXT}(y) \& \forall z(z \in y \equiv z=x))$

If $a$ is an object, let $F$ be the concept $[x: x=a]$. The requisite sentence is $\exists x(x=x)$. We have $\exists x(x=x)^{F}$ and $\forall G\left(\exists x(x=x)^{G} \rightarrow\right.$ $F \leq G)$. So $F$ is bounded and, by (RF), has an extension $\{a\}$ whose only member is $a$.

Pairs: $\forall x \forall y \exists z(\operatorname{EXT}(z) \& \forall w(w \in z \equiv(w=x \vee w=y)))$

If $a$ and $b$ are objects, let $F$ be the concept $[x: x=a \vee x=b]$. The requisite sentence is $\exists x \exists y(x \neq y)$, and, as usual, $\{a, b\}$ is the unordered pair of $a$ and $b$.

As usual, we define the ordered pair $\langle a, b\rangle$ of objects $a$ and $b$, to be $\{\{a\},\{a, b\}\}$. It is straightforward that $\langle a, b\rangle=\langle c, d\rangle$ if and only if $a=c$ and $b=d$.

Infinity: There is a Dedekind infinite extension.

FZBB2 in FZBB. The easiest way is to add a constant $c$ to the latter, with an axiom $\forall X \neg \operatorname{Ext}(c, X)$, stating that $c$ is not the extension of any concept. It is straightforward that the result is conservative over FZBB. Then interpret $\epsilon(X)=x$ as $(\operatorname{Ext}(x, X) \vee(x=c \& \neg \exists x \operatorname{Exт}(x, X)))$. 
The existence of the empty extension and pairs together entail the existence of $\emptyset,\{\emptyset\},\{\{\emptyset\}\}$, etc. By (RV) these are all different. So the universe is Dedekind infinite. Let $F$ be the least concept which applies to the empty set and is closed under the singleton operation. That is:

$$
\forall x(F x \equiv \forall X((X \emptyset \& \forall z(X z \rightarrow X\{z\})) \rightarrow X x)) .
$$

Let $\Phi$ be the following sentence, stating that the universe is Dedekind infinite:

$$
\exists f(\forall x \forall y(f x=f y \rightarrow x=y) \& \exists x \forall y(x \neq f y)) .
$$

Then we have $\Phi^{F} \& \forall G\left(\Phi^{G} \rightarrow F \leq G\right)$. So $F$ has an extension whose members are precisely $\emptyset,\{\emptyset\},\{\{\emptyset\}\}$, etc.

We do not have unrestricted versions of the other axioms of ZFC. In most cases, the reason is that FZBB2 provides only a sufficient condition for a concept to have an extension, namely, to be bounded. But we can derive restricted versions of the $\mathrm{ZF}$ axioms, and these do give the theory considerable power.

Bounded replacement: If $F$ is bounded and $G \leq F$ then $G$ is Good.

Bounded separation: If $F$ is bounded and $\forall x(G x \rightarrow F x)$ then $G$ is GooD.

On the limitation of size view, it would be natural to add a general principle of replacement: If $F$ is Good and $G \leq F$, then $G$ is Good. We do so at the start of next section.

Define the power-concept of a concept $F$, written $\mathcal{P}(F)$, to be the concept of being the extension of a subconcept of $F$. That is, $\mathcal{P}(F)$ is $[x: \exists X(x=\epsilon(X) \& \forall x(X x \rightarrow$ $F x)$ ]. It follows from bounded separation that if a concept $F$ is bounded, then every subconcept of $F$ is bounded and thus, by (RF), has an extension. So by (RV), every such subconcept has an extension. In the present context, the power set principle would be that if a concept is Good, then so is its power-concept. That does not follow from FZBB2, even if we add a general replacement principle. However, we do have:

Bounded power extension: If $F$ is bounded, then the power-concept of $F$ is Good.

So we have the existence of the power set of $\omega$, the power set of the power set of $\omega$, the power set of the power set of that, etc. And that is not the end. So FZBB2 has the resources to recapture virtually all of classical mathematics, except for foundational theories like ZFC, category theory, and the like. It is thus a reasonably powerful-if awkward - theory. By bounded replacement, we also have the existence of an extension that contains $V_{\omega}$ and is closed under power extensions. We will later make use of this fact in order to show that FZBB2 has the resources to prove the existence of a model for Zermelo set theory. 
Define the union-concept of a concept $F$, written $\bigcup F$, to be the concept of being a member of an extension of which $F$ holds: $\bigcup F$ is $[x: \exists y \exists X(y=\epsilon(X) \& F y \& x \in$ $y)$ )]. Recall that $x \in y$ only if $y$ is an extension. So the union-concept of $F$ is just $[x: \exists y(F y \& x \in y)]$. An unrestricted principle of unions would be that if $F$ has an extension, then so does the union-concept of $F$. As we will see in the next section, this does not hold in full generality here, alas. The best we can do is the following:

Doubly bounded union: Suppose that $F$ is bounded. Suppose further that there is a bounded concept $H$ such that for every $a$ such that $F a$, the concept $[x: x \in a]$ is smaller than or equinumerous with $H$. Then the union-concept of $F$ is GooD.

Concerning the axiom of choice, the best option seems to be to add a version of the axiom to the underlying higher-order logic, as a sort of general logical principle. One plausible candidate is in [13]:

$$
\forall R(\forall x \exists y R x y \rightarrow \exists f R x f x)
$$

The logical relationship between this and the more usual axiom of choice in set theory further highlights the fact that we do not have a non-trivial, necessary condition for a concept to be Good. In effect, (AC) is a global choice principle. Suppose, for example, that $F$ is a concept such that for every $x$, if $F x$ then $x$ is a non-empty extension and if $F x, F y$ and $x \neq y$, then $x$ and $y$ are disjoint. Then (AC) entails that there is a "choice concept", a concept $G$ such that for each $x$ such that $F x$, there is exactly one $y$ such that $y \in x$ and $G y$. We would like a local choice principle which says that if, in addition to the above, $F$ is Good then it has a Good choice concept. But, surprisingly, we do not have that. What we get from FZBB2 and (AC) is:

Bounded choice: Suppose $F$ is a bounded concept such that for every $x$, if $F x$ then $x$ is a non-empty extension and if $F x, F y$ and $x \neq y$, then $x$ and $y$ are disjoint. Then there is a GooD concept $G$ such that for each $x$ such that $F x$, there is exactly one $y$ such that $y \in x$ and $G y$.

As above, (AC) entails the existence of a choice-concept $G$ for $F$. But $G \leq F$ and so $G$ is bounded and thus Good.

For roughly the reasons provided by [14], FZBB2, even with (AC) and replacement, does not entail that the membership relation is well-founded. This contravenes the iterative conception of set, sketched above. The usual move, in contexts like this one, is to restrict attention to extensions generated by a certain process. One begins with the empty extension, and, at any given stage, one takes extensions of concepts under which only extensions generated thus far fall. The resulting extensions are hereditary well-founded extensions. They are hereditary because they have only extensions in their transitive closure; and they are well-founded because the membership relation on them is well-founded. 
We define this notion of hereditary well-founded extension, abbreviated $h w f$, by invoking a technique from [2], following [20]. ${ }^{14}$ Define a concept $F$ to be closed if:

$$
\forall y((\operatorname{EXT}(y) \& \forall z(z \in y \rightarrow F z)) \rightarrow F y)
$$

In words, $F$ is closed if, whenever it holds of the members of an extension, then it holds of that extension:

$$
h w f(x) \equiv_{d f} \forall F(\operatorname{closed}(F) \rightarrow F x) .
$$

In words, an object is hereditarily well-founded if and only if every closed property holds of it.

It is straightforward to show that the membership relation, restricted to $h w f$ extensions, is well-founded. In the context of New V, Boolos showed that $x$ is $h w f$ if and only if $x$ is an extension and every member of $x$ is $h w f$. The proof carries over to the general context here (see [28] 76-78). So the empty set is hereditary well-founded, and if $x$ and $y$ are $h w f$, then so is their pair, $\{x, y\}$. The von Neumann ordinals are all $h w f$, and, in particular, $\omega$ is $h w f$. If $x$ is $h w f$ and if the power set of $x$ exists, then it is $h w f$, etc. So the power set of $\omega$, the power set of that, the power set of that, etc., are all $h w f$. And if $x$ is $h w f$ and the union of $x$ exists, then it, too, is $h w f$.

Proposition 1 of [32] is that FZBB and so FZBB2 entails the satisfiability and thus consistency of Zermelo set theory, with choice. We show first that $V_{\omega}$, the set of all hereditarily finite sets, exists and is countable. By bounded power set, we then have $\mathcal{P}\left(V_{\omega}\right)$ exists, which is $V_{\omega+1}$. Similarly, we have the existence of $V_{\omega+2}, V_{\omega+3}$, etc. By bounded replacement, we have the existence of $\left\{V_{\omega+n}: n \in \omega\right\}$. The existence of $V_{\omega+\omega}$ is then a consequence of doubly bounded union. But $V_{\omega+\omega}$ is of course a standard model of Zermelo set theory (and FZBB2 can show this.) To show the consistency of the axiom of choice with Zermelo set theory, one can mimic Gödel's technique of constructibility.

The same techniques can be used to show more. ${ }^{15}$ The limits of these techniques are tied to the limits of the ability to define bounds in the pure second-order language: if $\alpha$ is a countable ordinal such that FZBB(2) entails that there is a GooD, well-ordered extension of order-type $\alpha$, then $\operatorname{FZBB}(2)$ entails the existence of $V_{\alpha}$. It should be clear, then, that $\mathrm{FZBB}(2)$ is a powerful theory of sets. The $h w f$ extensions make for a substantial universe, one sufficient to recapture almost all of contemporary mathematics, and then some.

\footnotetext{
${ }^{14}[2]$ uses the term "pure" for what we have called "hereditary well-founded". As [14] note, this can be misleading. Our theory has models in which there are extensions $e$ such that membership on $e$ is not well-founded, but the members of $e$, the members of the members of $e$, etc., are all extensions. Indeed, there can be an extension $e$ such that $e=\{e\}$.

${ }^{15}$ Thanks to Philip Welch for pointing this out to us.
} 


\section{What's not included}

Limits on the power of FZBB2 come from a study of its models. While we are at it, let us add an unrestricted replacement axiom, which is part and parcel of the limitation of size view which we used to motivate reflection in the first place:

$$
\forall X \forall Y((\operatorname{Good}(X) \& Y \leq X) \rightarrow \operatorname{Good}(Y)) .
$$

Unrestricted separation follows, and, FZBB2, (REP), and our global choice principle (AC) entails local choice.

The meta-theory here is ordinary first-order ZFC. The only primitive, non-logical terms in the object language are the higher-order predicate GooD and the extension operator $\epsilon$. To keep the present study in line with the corresponding $\$ 7$ of [32], we look at structures of the form $\mathcal{M}=\langle d, E\rangle$, where $d$ is a non-empty set, $E$ is a set of ordered pairs $\langle b, a\rangle$, where $b \in d$ and $a \subseteq d$.

The thought is that $d$ is the domain of interpretation of $\mathcal{M}$ and $\langle b, a\rangle \in E$ only if, in $\mathcal{M}, a$ is (in the extension of) Good and $\epsilon(a)=b$. We take it as written that there is one designated object $g \in d$ such that there is no Good subset $a \subseteq d$ with $\langle g, a\rangle \in E$. We take it that if $a$ is a BAD concept, then $\epsilon(a)=g$.

In the models we construct, the domain $d$ is a transitive set and, for each GooD concept $b$, the extension of $b$ is $b$ itself. That is, if $b$ is GooD, then $\epsilon(b)=b$, and so $\langle b, b\rangle \in E$. For this to be possible, of course, all of the Good concepts must themselves be members of the domain $d$.

In line with (REP), the Good concepts of each model are the subsets of its domain that are smaller than a certain, fixed cardinality. To specify one of our models, then, we give its domain $d$ and a cardinal number $\lambda$. So $E$ is $\{\langle x, x\rangle: x \in d \&|x|<\lambda\}$. These correspond to what [14] call $(\kappa-\lambda)$-models of the relevant variant of (RV).

For convenience, introduce a "dummy" symbol $\Omega$ for the concept of being a cardinal. If $\alpha$ is a cardinal, then just read $\alpha \in \Omega$ and $\alpha<\Omega$ as short for $\alpha=\alpha$. Let $\kappa$ be any cardinal or $\Omega$. For each sentence $\Phi$ of our pure second-order language, let $f(\Phi)$ be the smallest cardinal $\delta<\kappa$ such that $\Phi^{\delta}$ is true, if there is such a cardinal $\delta$; otherwise, let $f(\Phi)=0$. Thus

$$
f(\Phi)= \begin{cases}\delta & \text { if } \Phi^{\delta} \text { and for every } \gamma<\delta, \neg \Phi^{\gamma} \\ 0 & \text { if no such } \delta \text { exists. }\end{cases}
$$

In effect, $f(\Phi)$ is the cardinality of the smallest model of $\Phi$ that is itself smaller than $\kappa$, if there is such a model. Define the $\kappa$-limit, $l_{\kappa}$ to be the union of the set of all $f(\Phi)$. So if a sentence $\Phi$ is satisfiable on a set smaller than $\kappa$, then it is satisfiable on a set smaller than the $\kappa$-limit. ${ }^{16}$ Notice that since there are only countably many sentences in the language, if the cofinality of $\kappa$ is uncountable, then $l_{\kappa}<\kappa$.

\footnotetext{
${ }^{16}$ The $\Omega$-limit is called the "Löwenheim number" for the pure second-order language (see [30], 147-157, and the references cited there).
} 
Recall that a cardinal $\kappa$ is a strong limit if, for any cardinal $\lambda<\kappa, 2^{\lambda}<\kappa$. As Philip Welch pointed out, the following is a considerable strengthening of Proposition 4 is Section 7 of [32]:

Proposition 1. (ZFC). Let $\kappa$ be a strong limit of uncountable cofinality, and let $\lambda$ be any cardinal such that $l_{\kappa} \leq \lambda<\kappa$. Then there is a standard transitive model $\mathcal{M}=\langle d, E\rangle$ of FZBB2, (AC), foundation, and (REP) according to which a subset $a \subseteq d$ is GooD if and only if $|a|<\lambda$.

Proof Sketch. We need a set $d$ such that for any $a \subseteq d$, if $|a|<\lambda$, then $a \in d$. In other words, $d$ contains all of its subsets that are smaller than $\lambda$.

Consider the set $H C(\lambda)=\{x:|\operatorname{trcl}(x)|<\lambda\}$, which is the set of sets of cardinality hereditarily less than $\lambda$. Now: if $\lambda$ is regular, then $|H C(\lambda)|=\left|\bigcup_{\gamma<\lambda} 2^{\gamma}\right|$; if $\lambda$ is singular, then $|H C(\lambda)|=2^{\lambda}$. Either way, since $\kappa$ is a strong limit, we have that $|H C(\lambda)|<\kappa$ as desired.

The structure $\mathcal{M}$ we are looking for is $\langle d, E\rangle$, where $E$ is $\{\langle x, x\rangle: x \subseteq d \&|x|<$ $\lambda$ \}. As noted above, the defined membership relation, on the extensions of $\mathcal{M}$, coincides with the membership relation of the background meta-theory. So, $\mathcal{M}$ satisfies foundation. The satisfaction of (RV), (REP), and (AC) are immediate. That leaves only:

$$
\exists G\left(F \leq G \& \Phi^{G} \& \forall H\left(\Phi^{H} \rightarrow G \leq H\right)\right) \rightarrow \operatorname{GooD}(F)
$$

So let $\Phi$ be a sentence in the language of pure second-order logic. Suppose that there is a set $F \subseteq d$ such that $\mathcal{M}$ satisfies $\left(\exists G\left(F \leq G \& \Phi^{G} \& \forall H\left(\Phi^{H} \rightarrow G \leq H\right)\right)\right.$ ). We have to show that $F$ is Good, which amounts to $|F|<\lambda$. There is a subset $G$ of $d$ such that $F \leq G, \mathcal{M}$ satisfies $\Phi^{G}$, and $\mathcal{M}$ satisfies $\forall H\left(\Phi^{H} \rightarrow G \leq H\right)$ ). Because the quantifiers in $\Phi^{G}$ are all restricted, it is absolute in the structure. Since $\mathcal{M}$ is standard, so is the " $\leq$ " relation. So $\Phi^{G}$ is true, and for all subsets $H \subseteq d$, if $\Phi^{H}$ then $G \leq H$. Since $|d|<\kappa$, there is a cardinal $\delta<\kappa$ such that $\Phi^{\delta}$ is true. Recall that $f(\Phi)$ is the smallest such cardinal. Let $X$ be any subset of $d$ of cardinality $f(\Phi)$. Then $\Phi^{X}$ is true, and so $\mathcal{M}$ satisfies $\Phi^{X}$. So $G \leq X$ (indeed, $G \approx X$ ). Since $F \leq G$, we have that $F \leq X$, and so $|F| \leq f(\Phi)$. But $f(\Phi)<l_{\kappa}$ and $l_{\kappa} \leq \lambda$. So $|F|<\lambda$, and so, in $\mathcal{M}, F$ is Good. That is, $\mathcal{M}$ satisfies $\epsilon(F)=F$.

Recall that $\beth_{0}=\boldsymbol{\aleph}_{0}$; for each $\alpha, \beth_{\alpha+1}=\mathcal{P}\left(\beth_{\alpha}\right)$; and if $\lambda$ is a limit ordinal, then $\beth_{\lambda}=\bigcup\left\{\beth_{\alpha}: \alpha<\lambda\right\}$. Any limit in the $\beth$-series is a strong limit cardinal, and the cofinality of $\beth_{\lambda}$ is the cofinality of $\lambda$. So $\beth_{\omega_{1}}$ is a strong limit of uncountable cofinality. Thus from proposition 1, there is a standard model of FZBB2, (AC), foundation and (REP), whose domain is smaller than $\beth_{\omega_{1}}$. A fortiori, ZFC entails that our theory is consistent.

The Generalized Continuum Hypothesis (GCH) is that for every $\alpha, \boldsymbol{\aleph}_{\alpha}=\beth_{\alpha}$. So $\mathrm{ZFC}+\mathrm{GCH}$ entails that there is a standard transitive model of FZBB2, (AC), foundation and (REP), whose domain is smaller than $\boldsymbol{\aleph}_{\omega_{1}}$. Thus: 
Corollary 2. (ZFC) FZBB2, (AC), foundation, and (REP) does not deductively entail that there is a limit cardinal whose cofinality is uncountable.

Thus, by the standard set by ZFC, our theory is paltry.

Two more corollaries of Proposition 1 are that FZBB (together with (AC), foundation, and (REP)) does not entail the unrestricted union and power extensions principles. For the first, take $\kappa$ to be any strong limit of uncountable cofinality, or $\Omega$, and let $\lambda$ be the $\kappa$-limit $l_{\kappa}$. Then we obtain a model in which union fails rather badly. Because this $\lambda$ has countable cofinality, there is a countable series $\delta_{1}, \delta_{2}, \ldots$ such that each $\delta_{i}$ is a Good subset of the model, and so is its own extension, and thus is a member of the model. Moreover, the set $D=\left\{\delta_{1}, \delta_{2}, \ldots\right\}$ is also Good (since it is countable), but the union $\bigcup D$ is BAD since $|\bigcup D|=\kappa \cdot{ }^{17}$

Next, take $\kappa$ to be any strong limit of uncountable cofinality, or $\Omega$, and let $\eta$ be any cardinal such that $l_{\kappa}<\eta<\kappa$. Let $\lambda$ be $\eta^{+}$, the smallest cardinal greater than $\eta$. In the model that results from Theorem 1, let $a$ be any subset of the domain such that $|a|=\eta$. Then $a$ is GooD, and is thus a member of the domain. But the powerset of $a$ is of cardinality at least $\eta^{+}=\lambda$, and so it is BAD. ${ }^{18}$

In general, a model in the form of the conclusion of Proposition 1 satisfies the axioms of ZFC only if the indicated cardinal $\lambda$ is itself a strong inaccessible. In this case, $H C(\lambda)$ is just $V_{\lambda}$, which of course has cardinality $\lambda$. If $\lambda$ is any strong inaccessible, $V_{\lambda}$ is a model of FZBB2 only if there is another cardinal $\kappa>\lambda$ such that $l_{\kappa}<\lambda .{ }^{19}$

\section{Can we describe the indescribable?}

As noted, the above development, as it stands, is not particularly helpful to Scottish neo-logicism. To be sure, one of the axioms of FZBB2 is an abstraction principle, namely:

$$
\epsilon(F)=\epsilon(G) \equiv((\operatorname{BAD}(F) \& \operatorname{BAD}(G)) \vee \forall x(F x \equiv G x),
$$

but this is only a principle of extensionality. Recall that GooD is just a primitive in the language (with $\operatorname{BAD}(F)$ defined as $\neg \operatorname{Good}(F)$ ). The power of the theory comes from the reflection schema:

$$
\exists G\left(F \leq G \& \Phi^{G} \& \forall H\left(\Phi^{H} \rightarrow G \leq H\right)\right) \rightarrow \operatorname{GooD}(F),
$$

\footnotetext{
${ }^{17}$ See [32], Corollary 5, for details.

${ }^{18}$ Again, see [32], Corollary 7, for details.

${ }^{19}$ In [32], we stated that it is straightforward to verify that every standard model of FZBB is a strong inaccessible that is greater than the Löwenheim number for second-order logic. Unfortunately, it is not straightforward to verify this, since the conclusion is actually independent of ZFC.
} 
and this is not even close to an abstraction. In addition, (RF) only gives a sufficient condition for a concept to be Good and thus have an extension. Abstraction principles are to give necessary and sufficient conditions for the existence of the abstracts in question.

Recall that a concept $F$ is fixed by a sentence $\Phi$ just in case $\Phi^{F}$ and for every concept $X$, if $\Phi^{X}$ then $F \leq X$. And $F$ is bounded if there is a sentence $\Phi$ of pure second-order logic and a concept $G$ such that $F \leq G$ and $G$ is fixed by $\Phi$.

The theme of (RF) is that if a concept $F$ is bounded by a sentence of the pure second-order language, then $F$ is Good, and thus has an extension. A natural move for a neo-logicist, perhaps, would be to try to turn this into a necessary and sufficient condition, with a principle saying that a concept is GooD if and only if it is bounded by a sentence of the pure second-order language. If this could be expressed, there would be an explicit definition of Good (and thus BAD), and so (RV) could be rendered as a full-fledged abstraction principle.

The obvious route to such an explicit definition is blocked. Indeed, suppose that there were a formula $\Psi(X)$ in the pure second-order language such that for each concept $F, \Psi(F)$ if and only if $F$ is bounded. Then there would be a sentence that fixes the smallest unbounded concept, the Löwenheim number for the pure second-order language. But if there were a concept that large, then it, too, would be bounded, which is impossible.

This is only to be expected. We began with the inchoate thought, shared by many set theorists, that the universe is ineffable, and we took that to be something like "not characterized by logical resources alone". And "logical resources" was glossed as something like "bounded by a sentence of pure second-order logic". So we cannot expect to be able to describe the universe using those same logical resources. One cannot describe the indescribable.

To be sure, our notion of "bounded" is definable in ordinary set theory. That is why we were able to characterize models of the theory in the previous section, within ZFC. But the neo-logicist is out to found or reconstruct set theory, and so cannot simply use it.

One option is to ascend to a third-order language. Let $\mathcal{E}$ be a variable ranging over concepts of concepts. There is a formula $D E F(\mathcal{E})$ of "pure" third-order logic that is satisfied by $\mathcal{E}$ if and only if $\mathcal{E}(X)$ is satisfied by a concept $F$ if and only if there is a sentence of pure second-order logic such that $\Phi^{F}$.

It is not difficult, though tedious, to construct $D E F$ by mimicking a Tarskian explicit definition of truth for the second-order fragment of the language. We have that a concept $F$ is fixed if and only if:

$$
\exists \mathcal{E}(D E F(\mathcal{E}) \& \mathcal{E} F \& \forall Y(\mathcal{E} Y \rightarrow F \leq Y),
$$


and $F$ is bounded if and only if

$$
\exists X(F \leq X \& \exists \mathcal{E}(D E F(\mathcal{E}) \& \mathcal{E} F \& \forall Y(\mathcal{E} Y \rightarrow F \leq Y) .
$$

Call this last formula $\operatorname{BAD}^{2}(F)$, and define $\operatorname{GooD}^{2}(F)$ as $\neg \mathrm{BAD}^{2}(F)$. Now we do have the resources to say that a concept has an extension if and only if it is bounded:

$$
\epsilon(F)=\epsilon(G) \equiv\left(\left(\mathrm{BAD}^{2}(F) \& \mathrm{BAD}^{2}(G)\right) \vee \forall x(F x \equiv G x) \quad\left(\mathrm{RV}^{3}\right)\right.
$$

This $i$ an abstraction principle. So if our neo-logicist can invoke a third-order language, he can at least potentially use $\left(\mathrm{RV}^{3}\right)$ to ground a mathematical theory. And it is a fairly powerful theory of sets. By comprehension, each instance of (RF) is a consequence of $\left(\mathrm{RV}^{3}\right)$. So the results from previous sections carry over, and some of them can be sharpened. The theory $\left(\mathrm{RV}^{3}\right)$ entails the principles of empty extension, singletons, pairs, infinity, doubly-bounded union, and unrestricted replacement. As above, the local axiom of choice follows from (AC), and the axiom of foundation holds on the hereditarily well-founded extensions. And there is more. Since every Good ${ }^{2}$ concept is bounded, we also have an unrestricted power-extension principle: if a concept $F$ has an extension, then so does its powerconcept. So $\left(\mathrm{RV}^{3}\right)$ entails all of the axioms of ZFC, except for union.

However, the omission of (unrestricted) union from the consequences of $\left(\mathrm{RV}^{3}\right)$ cannot be easily remedied, say by adding it as another axiom. Indeed, the negation of even a weak union principle is a logical consequence of $\left(\mathrm{RV}^{3}\right)$. Extensions of the results of the previous section show that for each standard model of $\left(\mathrm{RV}^{3}\right)$, foundation, and (AC), there is a cardinal $\kappa$ (or $\Omega$ ) such that a subset $a$ of the domain is GooD ${ }^{2}$ if and only if $|a|<l_{\kappa}$, where $l_{\kappa}$ is the $\kappa$-limit (as defined in the previous section). And recall that the $\kappa$-limits all have cofinality $\omega$. So it follows that in each standard model there is a countable set $a=\left\{a_{1}, a_{2}, \ldots\right\}$, such that $a$ is GooD ${ }^{2}$ (by replacement, because $a$ is countable), each $a_{i}$ is GooD (because it is smaller than $l_{\kappa}$ ), but the union of $a$ is $\mathrm{BAD}^{2}$ (because it has size $l_{\kappa}$ ). In other words, each standard model of $\left(\mathrm{RV}^{3}\right)$, foundation, and $(\mathrm{AC})$ has a counterexample to even countable union. Moreover, in each standard model of $\left(\mathrm{RV}^{3}\right)$ the concept of being a hereditarily well-founded extension will have confinality $\omega$.

Nevertheless, $\left(\mathrm{RV}^{3}\right)$ is a powerful theory. Since it entails the axioms of FZBB2 (and FZBB of [32]), it has the resources to reconstruct every extant mathematical theory short of foundational theories like ZFC and the various category theoretic foundations. It has a model of Zermelo set theory, and much more. So perhaps it can be deployed by the neo-logicist, as an awkward but reasonably powerful theory of sets.

\section{The Company you keep}

We presume that readers of this volume do not need a detailed overview of the Bad Company objection to Scottish neo-logicism. In a nutshell, the complaint is 
that abstraction principles cannot be a legitimate way to introduce mathematical theories, since some abstractions are inconsistent. The most notorious example, of course, is Frege's own Basic Law V:

$$
\epsilon(F)=\epsilon(G) \equiv \forall x(F x \equiv G x)
$$

As Michael Dummett, a prominent critic of the program, concedes, the Bad Company objection is better seen as a challenge: "Possibly some restriction, distinguishing the case of cardinal numbers from that of value-ranges could be framed." ${ }^{20}$ One rather obvious requirement is that an acceptable abstraction principle should be consistent. But this is not sufficient, as there are abstraction principles that are satisfiable only on finite domains. Such principles conflict with Hume's principle.

The Scottish neo-logicists have risen to this challenge, and proposed a number of conservation requirements on acceptable abstractions. Many of these can be formulated, external to the program, in model-theoretic terms. Say that an abstraction $\Sigma$ is stable $e^{21}$ if there is some cardinal $\lambda$ such that for all $\kappa>\lambda, \Sigma$ is satisfiable in a domain of cardinality $\kappa$. This is a rather stringent requirement and perhaps a desirable quality for a proposed abstraction principle to enjoy. If $\Sigma$ is stable, then given a sufficiently large initial domain, there must be a way to interpret the abstraction operator on it so that $\Sigma$ is true. One more reason to think stability is desirable is the observation that given the axiom of choice, Hume's principle, which is the neo-logicist gold standard, is stable.

Crispin Wright once made the following proposal: "Why not just say that pairwise incompatible but individually conservative abstractions are ruled out . . . and have done with it" (assuming a precise definition of conservativeness, which is omitted here). ${ }^{22}$ This suggests yet another criterion for acceptability. Say that an abstraction principle $\Sigma$ is irenic if it is jointly satisfiable with every conservative abstraction principle. Remarkably, [37] shows that if an abstraction principle is stable, then it is irenic. ${ }^{23}$

Thus stability may initially seem an attractive necessary and sufficient condition for acceptability. ${ }^{24}$ We must report, however, that the present $\left(R V^{3}\right)$ is not stable.

\footnotetext{
${ }^{20}$ See [6], p. 375.

${ }^{21}$ As far as we know, the notion of stability was first formulated by [12], n. 5, who called it a "promising necessary condition on" abstractions. [7], 511, and [8], 114, seems to be the first to employ the word "stable" for this criterion.

${ }^{22}$ See [38], 23.

${ }^{23}$ See his Theorem 6.1. [37] invokes a slightly stronger notion, defining an abstraction $\Sigma$ to be stable "if for some cardinal $\lambda, \Sigma$ is true at all and only models of cardinality $\geq \lambda$ (emphasis ours). He shows that if an abstraction is stable in that sense, then it is irenic. Unfortunately, the converse does not hold for the stronger notion, despite the statement of Weir's Theorem 6.1. His argument for this actually invokes the present notion, and the result is correct for it. Weir's result assumes that the background language is at least third-order, since it involves replacing an abstraction operator with a variable and then binding that variable.

${ }^{24}$ Though see [21] for arguments to the effect that stability alone cannot be sufficient for accept-
} 
This follows from a more general result. In present terms, say that a theory of sets satisfies "countable replacement" if it entails that every countable concept is GooD. This is a rather minimal requirement, one that just about any set theory should satisfy (although Zermelo set theory does not). $\left(\mathrm{RV}^{3}\right)$ easily satisfies countable replacement, since it satisfies unrestricted replacement and infinity. So does FZBB2, since that theory satisfies bounded replacement and infinity.

[34] shows that no set theory that satisfies countable replacement is stable. In particular, such a theory is not satisfiable at a cardinal $\kappa$ if $\kappa$ has more than $\kappa$-many countable subsets. It is easy to see that there are unboundedly many such cardinals. Indeed if $\lambda$ is a limit ordinal with confinality $\omega$ (e.g., if $\lambda=\kappa+\omega$, for any cardinal $\kappa)$, then $\beth_{\lambda}$ has more than $\beth_{\lambda}$-many countable subsets. In particular, $\left(\mathrm{RV}^{3}\right)$ (and FZBB2) has no model of size $\beth_{\lambda}$.

It seems that a neo-logicist who hopes to capture a decent set theory cannot adopt stability as a necessary condition on abstraction principles. ${ }^{25}$

This might not be as serious as it sounds. Set theory is supposed to be a foundational theory, able to provide surrogates for any mathematical objects whatsoever. It is to describe the realm of models for itself and other theories. So it is no wonder that there are arbitrarily large sets on which set theory itself fails to be satisfiable. Perhaps our neo-logicist can accept $\left(\mathrm{RV}^{3}\right)$ by pleading that a foundational theory need not be stable. This leaves open a Bad-Company-style objection from the possibility of an alternative acceptable foundational theory, which fails to be stable but which is incompatible with $\left(\mathrm{RV}^{3}\right)$. This would be an analogue of the "distraction principles" in [37]. But we will not pursue this line any further.

Say that an abstraction principle $\Sigma$ is unbounded if, for every cardinal $\lambda$, there is a $\kappa>\lambda$ such that $\Sigma$ has a model whose domain has cardinality $\kappa$. So if $\Sigma$ is unbounded, then (assuming the axiom of choice) we can turn any set into a model of $\Sigma$ by adding more elements (presumably to serve as abstracts).

It is reasonable to require that any acceptable abstraction principle—especially a foundational one-be unbounded. For one reason, an abstraction principle is conservative, in one of the relevant senses, if and only if it is unbounded. More telling perhaps, is the observation that a bounded abstraction principle imposes an upper bound to the size of the universe. Recall that, according to Scottish neo-logicism, acceptable abstraction principles are supposed to be the sort of thing that can be known a priori, as a matter of definitional stipulation. If an acceptable abstraction were bounded, then it would be possible to know a priori, as a matter of stipulation, that the universe is smaller than a given, fixed cardinal $\kappa$. If nothing else, neo-logicism would be incompatible with the meta-theory in which the notion of

${ }^{25}$ [34] does show that second-order Zermelo set theory, with urelements, is stable. Recall that Zermelo set theory does not satisfy replacement. It does satisfy union, of course. In a sense, $\left(\mathrm{RV}^{3}\right)$ is the opposite, since it satisfies replacement but not union.
} 
boundedness is formulated-the theory in which the existence of $\kappa$ is formulated and proved.

The good news is that $\left(\mathrm{RV}^{3}\right)$ is unbounded. Recall our definition of the $\Omega$-limit, sometimes called the Löwenheim number for second-order languages: for each sentence $\Phi$ of our pure second-order language, let $f(\Phi)$ be the smallest cardinal $\delta$ such that $\Phi^{\delta}$ is true, if there is such a cardinal $\delta$; otherwise, let $f(\Phi)=0$. The $\Omega$-limit, $l_{\Omega}$ is the union of the set of all $f(\Phi)$. So if a sentence $\Phi$ is satisfiable at all, then it is satisfiable on a set smaller than the $\Omega$-limit. Let $\lambda$ be the $\Omega$-limit, and let $\kappa$ be any cardinal. It is easy to check that there is a set $d$ such that $|d|>\kappa$, and for any $a \subseteq d$, if $|a|<\lambda$ then $a \in d$. Such a set $d$ is a model of $\left(\mathrm{RV}^{3}\right)$.

We must report, however, that this bit of good news is not really all that good. Even though there is no upper limit to cardinality to the domain of models of $\left(\mathrm{RV}^{3}\right)$, the theory does fix an absolute upper bound on the size of GooD ${ }^{2}$ concepts, namely the Löwenheim number $\lambda$. There is no model of $\left(\mathrm{RV}^{3}\right)$ which contains an extension with $\lambda$-many members. This is, admittedly, a rather large bound, at least by any standards other than those of a jaded set theorist. If there is an inaccessible cardinal, then $\lambda$ is larger than the smallest one; if there is a measurable cardinal, then $\lambda$ is larger than the smallest one, etc. ${ }^{26}$

Nevertheless, $\left(\mathrm{RV}^{3}\right)$ does set an upper bound to the size of GooD ${ }^{2}$ concepts and thus extensions. And if our set theory is to play its foundational role, it is the extensions that are to provide surrogates for mathematical objects. So if $\left(\mathrm{RV}^{3}\right)$ is an acceptable abstraction principle, and is to play its foundational role, then we would know, a priori, that there are no more than $\lambda$ mathematical objects. This is in obvious tension with ZFC, the very theory in which we establish this bound. We do not speculate as to how this tension should be resolved.

\section{Ineffability again}

The real conceptual problem with $\left(\mathrm{RV}^{3}\right)$ is that it is itself in tension with the thesis that the universe of extensions is ineffable-the very heuristic hypothesis that motivates our development of FZBB2 (and FZBB of [32]). Since the initial background language for our theorizing was second-order, we glossed, or explicated, the inchoate notion of ineffability as something like "not bounded by a sentence of the pure second-order language", and we adopted a scheme that makes being bounded

\footnotetext{
${ }^{26}$ A property $P(x)$ of sets is said to be "local" if there is a formula $\Psi(x)$ in the language of firstorder set theory, such that for each $x, P(x)$ holds if and only if $\exists \delta\left(V_{\delta} \mathcal{M}\right.$ odels $\left.\Psi(x)\right)$ (where $V_{\delta}$ is the $\delta$ th rank). The idea is that local properties are those with a characterization that only refers to the sets below a fixed rank. One does not need to refer to "arbitrarily large" sets in order to state whether a given set has the property. Inaccessible, Mahlo, hyper-Mahlo, and measurable are all local properties. Define a cardinal $\lambda$ to be "minimal-local" if there is a local property $P(x)$ such that $\lambda$ is the smallest cardinal with property $P(x)$. The $\Omega$-limit is the union of all minimal-local cardinals. So, for example, if there is a measurable cardinal, then the $\Omega$-limit is greater than the smallest one.
} 
by a sentence of that language a sufficient condition for a concept to be Good. We obtained some mileage from that, by bootstrapping. But now, with $\left(\mathrm{RV}^{3}\right)$, we move to a third-order language and thereby manage to say exactly which concepts are not bounded by a sentence of the pure second-order language, making that a necessary and sufficient condition for a concept to have an extension. But if the thought that the universe is ineffable is correct, as explicated above, then it should also entail that the universe cannot be bounded by a sentence of the pure third-order language either. And $\left(\mathrm{RV}^{3}\right)$ violates that, as it does give a bound to the size of the GooD concepts (and this bound is definable in the third-order language).

Once we move to a third-order language, the hypothesis of ineffability should be glossed, or explicated, as something like "not bounded by a sentence of the pure third-order language", and this would motivate a scheme that makes being bounded by a sentence of the pure third-order language a sufficient condition for a concept to be GooD. Let $\left(\mathrm{RF}^{3}\right)$ be the extension the scheme (RF) to include sentences from the pure third-order language, and let $\mathrm{FZBB}^{3}$ be $\left(\mathrm{RF}^{3}\right)$ plus $(\mathrm{RV})$. Then the entire development from $\S \S 4-6$ above can be recapitulated by replacing phrases like "pure second-order" with "pure third-order". Even the meta-theory carries over, almost word for word. The various $\kappa$-limits will be higher, of course (since are now dealing with third-order boundedness), but the results are completely analogous.

Needless to say, with $\mathrm{FZBB}^{3}$ now we are back to having only a sufficient condition for a concept to be GooD, and this is not particularly helpful to Scottish neo-logicism, for the above reasons. The problem is that the third-order language cannot characterize which concepts are bounded by sentences of that language. We could remedy that with an explicit definition in a fourth-order language, producing a single abstraction principle, which we can call $\left(\mathrm{RV}^{4}\right)$. But once we do that, the inchoate thought of ineffability suggests that the universe is not bounded by a sentence that language either. ${ }^{27}$

It can go on, even into the transfinite. Our standard meta-theory ZFC entails the consistency of each of the theories $\mathrm{FZBB}^{3}, \mathrm{FZBB}^{4}, \ldots, \mathrm{FZBB}^{\omega}, \ldots$, up to the limits of what can be expressed in the language of ZFC.

What we have here, it seems, an instance of the phenomenon that started the process of reflection in the first place. Recall how [36] 555, put it:

Any time we try to capture the universe from what we positively possess (or can express) we fail the task and the characterization is satisfied by certain (large) sets.

\footnotetext{
${ }^{27}$ As stated, $\left(\mathrm{RV}^{4}\right)$ is incompatible with $\left(\mathrm{RV}^{3}\right)$. In particular, $\left(\mathrm{RV}^{4}\right)$ will declare some concepts to be Good that $\left(\mathrm{RV}^{3}\right)$ declares to be BAD. That is because the $\Omega$-limit (or Löwenheim number) for third-order languages is larger than the $\Omega$-limit for second-order languages. In other words, the two abstraction principles give incompatible necessary and sufficient conditions for a concept to be GooD. This is not a serious problem, since the neo-logicist can just use different $\epsilon$-operators in the different principles. As above, however, it is a serious problem if the neo-logicist wants to declare both theories to be foundational.
} 
Every time we add new expressive resources, we can establish the existence of extensions larger than any we could envision before. But we cannot think that we have it all. The very act of thinking about what we have—so far-gives us more than we think.

There is a marked trend in the mathematics of at least the past 150 years of not tying what exists to what can be constructed, defined, or otherwise characterized by this or that batch of expressive resources. The acceptance of the law of the law of excluded middle, non-constructive reasoning generally, impredicative definitions, the axiom of choice, and, more recently, the almost universal rejection of Gödel's principle of constructibility $V=L$, are all instances of this trend. ${ }^{28}$ If any of the theories in the form $\left(\mathrm{RV}^{\mathrm{n}}\right)$, or even $\left(\mathrm{RV}^{\alpha}\right)$ for some definable ordinal $\alpha$, were taken to be foundational, it would be a clear violation of this trend. The theory would declare that all and only extensions are bounded by sentences of the $n$ th-order, or $\alpha$ th-order, language.

The problem, we submit, applies to any neo-logicist attempt to recapture set theory, at least if the theory is to be foundational and proceed through a restricted version of Basic Law V, such as our (RV):

$$
\epsilon(F)=\epsilon(G) \equiv((\operatorname{BAD}(F) \& \operatorname{BAD}(G)) \vee \forall x(F x \equiv G x))
$$

Suppose that a neo-logicist comes up with a necessary and sufficient condition for a concept to be good, using whatever resources are allowed in acceptable abstraction principles. Call the definition Good. Then the abstraction principle would be:

$$
\epsilon(F)=\epsilon(G) \equiv((\neg \operatorname{Good}(F) \& \neg \operatorname{Good}(G)) \vee \forall x(F x \equiv G x))
$$

Suppose also that our neo-logicist establishes, or just adopts, a general replacement principle (in line with the limitation of size conception):

$$
\forall X \forall Y((\operatorname{Good}(X) \& \operatorname{Good}(Y) \& Y \leq X) \rightarrow \operatorname{Good}(Y))
$$

In words, if a concept is Good, then so is any concept of that size or smaller.

If this is accomplished, then the neo-logicist can give a bound to the universe, in the sense that she has the resources to specify how large the smallest $\mathcal{B a d}$ concept is. So she cannot adopt the foregoing theme of ineffability, that if a concept is bounded by whatever resources are in play, then it is Good, nor can she adopt the common view, articulated by Wang, that underlies reflection principles. If an abstraction principle, in the above form, is to have the foundational role played by set theory, then it would be a declaration that we can "capture the universe from what we positively possess (or can express)".

\footnotetext{
${ }^{28}$ Of course, this is not to say that there is something illegitimate with intuitionistic systems, predicative analyses, or the like. It is only to say that mathematics is not limited to such things.
} 


\section{Acknowledgements}

We are grateful to audiences at the final Abstraction Workshop at Arché (Status Belli) and at the Mathematical Methods in Philosophy in Banff, where earlier versions of this paper were presented. Thanks to Timothy Bays, John Burgess, Øystein Linnebo, Marcus Rossberg and Crispin Wright for helpful comments and discussion. We are especially grateful to Philip Welch for pointing out to us how to improve some of our previous results.

\section{References}

[1] Aldo Antonelli and Robert May. "Freges Other Program". Notre Dame Journal of Formal Logic, 46:117, 2005.

[2] George Boolos. "Iteration again". Philosophical Topics, 17:521, 1989.

[3] George Boolos. "The iterative Concepcion of Set". Journal of Philosophy, 68:215 231, 1971.

[4] John Burgess. "E Pluribus Unum: Plural Logic and Set Theory". Philosophia Mathe- matica (3), 12:13221, 2004.

[5] John Burgess. Fixing Frege. Princeton University Press, 2005.

[6] Michael Dummett. Frege: Philosophy of Mathematics. Harvard University Press, Cambridge, MA, 1991.

[7] Kit Fine. The Limits of Abstraction. 1998. In M. Schirn (ed.) The Philosophy of Mathematics Today. Clarendon Press, 503-629.

[8] Kit Fine. The Limits of Abstraction. Oxford University press, Oxford, 2002. 28

[9] A. Fraenkel, Y. Bar-Hillel, and A. Lévy. Foundations of Set Theory. North Holland, 1973.

[10] Bob Hale. "Abstraction and Set Theory". Notre Dame Journal of Formal Logic, 41 (4):379398, 2000a.

[11] Bob Hale. "Reals by Abstraction". Philosophia Mathematica (3), 8:100123, 2000b.

[12] Richard Heck. "On the Consistency of Second-Order Contextual Definitions". Noûs, 26:4914, 1992.

[13] David Hilbert and Wilhelm Ackermann. Die Grundlagen der theoretischen Logik. Springer, 1928. 
[14] Ignacio Jané and Gabriel Uzquiano. "Well- and Non-Well-Founded Fregean Extensions". Journal of Philosophical Logic, 33(5):437465, October 2004.

[15] Thomas J. Jech, editor. Axiomatic Set Theory, volume 13 of Proceedings of the Symposia on Pure Mathematics. American Mathematical Society, Providence, R.I., 1974.

[16] Thomas J. Jech. Set Theory. Springer, 3rd edition, 2006.

[17] Akihiro Kanamori. "The Mathematical Development of Set Theory from Cantor to Cohen”. Bulletin of Symbolic Logic, 2(1):171, 1996.

[18] Akihiro Kanamori. The Higher Infinite: Large Cardinals in Set Theory from their Beginnings. Springer, 2003.

[19] Peter Koellner. "On Reflection Principles". Annals of Pure and Applied Logic, 157: 206-219. February 2009.

[20] Azriel Lévy. "On von Neumanns Axiom System for Set Theory”. TheAmerican Mathematical Monthly, 75(7):762763, 1968.

[21] Øystein Linnebo and Gabriel Uzquiano. "Which Abstraction Principles Are Acceptable? Some Limitative Results" The British Journal for the Philosophy of Science, 60: 2, 239-252, June 2009.

[22] Dimitry Mirimanoff. "Les Antinomies de Russell et de Burali-Forti et le probléme Fondamental de la Theórie des Ensembles". LEnseignment Mathématique, 19: 37-52, 1917.

[23] Richard Montague. "Semantic Closure and Non-Finite Axiomatizability I". In Infinitistic Methods: Proceedings of the Symposium on the Foundations of Mathematics, 4569, New York, 1961. Pergamon.

[24] Michael Potter. Set Theory and Its Philosophy: A Critical Introduction. Oxford University Press, 2004.

[25] William Reinhardt. "Remarks on Reflection Principles, Large Cardinals, and Elementary Embeddings". 1974. In Thomas Jech, (ed.) Axiomatic Set Theory, vol. II of Proceedings of the Symposia on Pure Mathematics, American Mathematical Society, 189-205, 1974.

[26] M. Schirn (ed.) The Philosophy of Mathematics Today. Clarendon Press, 1998.

[27] Dana Scott. "Axiomatizing Set Theory. In Thomas J. Jech, editor, Axiomatic Set Theory, volume II of Proceedings of the Symposia on Pure Mathematics, American Mathematical Society, 20714,1974.

[28] Stewart Shapiro. "Prolegomenon to Any Future Neo-Logicist Set Theory: Abstraction and Indefinite Extensibility". British Journal for the Philosophy of Science, 54: 5991, 2003. 
[29] Stewart Shapiro. "Frege meets Dedekind: A Neo-Logicist Treatment of Real Analysis". Notre Dame Journal of Formal Logic, 41(4): 33564, 2000.

[30] Stewart Shapiro. Foundations Without Foundationalism: A Case for SecondOrder Logic. Clarendon Press, Oxford, 1991.

[31] Stewart Shapiro. "Principles of Reflection and Second-Order Logic". Journal of Philosophical Logic, 16:309333, 1987.

[32] Stewart Shapiro and Gabriel Uzquiano. "Frege meets Zermelo: A Perspective on Ineffability and Reflection". The Review of Symbolic Logic, 1(241-266), 2008 .

[33] William Tait. "Constructing Cardinals from Below". In William Tait (ed.), The Provenance of Pure Reason: Essays in the Philosophy of Mathematics and Its History. Oxford University Press, 2005.

[34] Gabriel Uzquiano. "Bad Company Generalized". Synthese, 170: 331-347. October 2009.

[35] John von Neumann. Über eine Widerspruchfreiheitsfrage in der axiomaticschen Mengenlehre. Journal für die reine und angewandte Mathematik (Crelles Journal), 160: 227241, 1929.

[36] Hao Wang. From Mathematics to Philosophy. Routledge \& Kegan Paul, 1974. 30

[37] Alan Weir. "Neo-Fregeanism: An Embarrassment of Riches". The Notre Dame Journal of Formal Logic, 44(1):1348, 2003.

[38] Crispin Wright. "Is Hume's Principle Analytic" Notre Dame Journal of Formal Logic, 40:1, 6-29, Winter 1999.

[39] Ernst Zermelo. Über Grenzzahlen und Mengenbereiche: Neue Untersuchungen über die Grundlagen der Mengenlehre. Fundamenta Mathematicae, 16: 2947, 1930. 